\title{
Fault diagnosis in industrial systems based on blind source separation techniques using one single vibration sensor
}

\author{
V.H. Nguyen, C. Rutten and J.-C. Golinval* \\ Structural Dynamics Research Group, Aerospace \& Mechanical Engineering Department, University of Liege, \\ Chemin des Chevreuils, Belgium
}

\begin{abstract}
In the field of structural health monitoring or machine condition monitoring, most vibration based methods reported in the literature require to measure responses at several locations on the structure. In machine condition monitoring, the number of available vibration sensors is often small and it is not unusual that only one single sensor is used to monitor a machine. The aim of this paper is to propose an extension of fault detection techniques that may be used when a reduced set of sensors or even one single sensor is available. Fault detection techniques considered here are based on output-only methods coming from the Blind Source Separation (BSS) family, namely Principal Component Analysis (PCA) and Second Order Blind Identification (SOBI). The advantages of PCA or SOBI rely on their rapidity of use and their reliability. Based on these methods, subspace identification may be performed by using the concept of block Hankel matrices which make possible the use of only one single measurement signal. Thus, the problem of fault detection in mechanical systems can be solved by using subspaces built from active principal components or modal vectors. It consists in comparing subspace features between the reference (undamaged) state and a current state. The angular coherence between subspaces is a good indicator of a dynamic change in the system due to the occurrence of faults or damages. The robustness of the methods is illustrated on industrial examples.
\end{abstract}

Keywords: Fault diagnosis, damage detection, vibration monitoring, Hankel matrices

\section{Introduction}

Separating a set of signals from their observed mixture can be realized using Blind Source Separation (BSS) techniques, even without any knowledge of the sources signals or the mixing process. Some methods in the BSS family are attractive, namely Principal Component Analysis (PCA), Independent Component Analysis (ICA) and Second-Order Blind Identification (SOBI). Their advantages rely on the versatility and the simplicity of practical use. However, a main drawback of BSS techniques is the need of several sensors. If the number of sensors is too small, modal analysis may not be performed in good conditions.

For the purpose of improving damage detection, an alternative PCA-based method named Null Subspace Analysis (NSA) which relies on the definition of block Hankel matrices was proposed and applied in [1] on the example of an airplane mock-up. The use of Hankel matrices was also exploited in [2] to enhance the detection of nonlinearity onset in a nonlinear system using Kernel Principal Component Analysis (KPCA). Based on the concept of block Hankel matrices, both methods (NSA and KPCA) showed their efficiency even when the number of sensors is small.

The aim of this paper is to present some examples of applications of output-only detection techniques in order to perform fault diagnosis in industrial systems. The paper is organized as follows. The two methods (PCA and SOBI)

*Corresponding author: J.-C. Golinval, Structural Dynamics Research Group, Aerospace \& Mechanical Engineering Department, University of Liege, Chemin des Chevreuils, 1 B52/3, B-4000 Liège 1, Belgium. E-mail: JC.Golinval@ulg.ac.be. 
which are considered as basic approaches are first described briefly. Then, the definition of block Hankel matrices is recalled and the enhanced methods (named EPCA and ESOBI) are introduced. The detection indicator which is considered here is based on the concept of subspace angle as proposed in [1]. Regarding to [1], this paper generalizes the use of Hankel matrices for other methods than PCA-based methods and illustrates the procedure of fault detection from just a single sensor on industrial applications which consist in detecting faults in a rotating device and damages during the welding process of joints.

\section{Principal component analysis (PCA)}

Let us consider a dynamical system characterized by a set of vibration measurements collected in the observation matrix $\mathbf{X} \in \mathfrak{R}^{m \times N}$, where $m$ is the number of sensors and $N$ is the number of time samples. Principal component analysis (PCA), also known as Karhunen-Loève transform or Proper Orthogonal Decomposition (POD) [3], provides a linear mapping of the data from the original dimension $m$ to a lower dimension $p$. The dimension $p$ corresponding to the number of principal components defines the order of the system. In practice, PCA is often performed by singular value decomposition (SVD) of matrix $\mathbf{X}$, i.e.

$$
\mathbf{X}=\mathbf{U} \Sigma \mathbf{V}^{\mathrm{T}}
$$

where $\mathbf{U}$ and $\mathbf{V}$ are orthonormal matrices, the columns of $\mathbf{U}$ defining the principal components (PCs). The order $p$ of the system is determined by selecting the first $p$ non-zero singular values in $\Sigma$ which have a significant magnitude ("energy") as described in [3]. A threshold in terms of cumulated energies is often fixed to select the effective number of PCs that is necessary for a good representation of matrix $\mathbf{X}$. In practice, a cumulated energy of $70 \%$ to $95 \%$ is generally adequate for the selection of the active PCs.

\section{Second order blind identification (SOBI)}

Second-order blind identification was introduced by Belouchrani et al. [4]. Like other BSS approaches, SOBI considers observed signals $\mathbf{x}(t)$ as a noisy instantaneous linear mixture of source signals $\mathbf{s}(t)$. In many situations, multidimensional observations are represented as:

$$
\mathbf{x}(t)=\mathbf{y}(t)+\boldsymbol{\sigma}(t)=\mathbf{A s}(t)+\boldsymbol{\sigma}(t)
$$

where, $\mathbf{x}(t)=\left[x_{1}(t), \ldots, x_{m}(t)\right]^{T}$ is a linear instantaneous mixture of source signals and of noise.

$$
\begin{aligned}
& \mathbf{s}(t)=\left[s_{1}(t), \ldots, s_{p}(t)\right]^{T} \text { contains the signals issued from } p \text { narrow band sources, } p<m . \\
& \mathbf{y}(t)=\left[y_{1}(t), \ldots, y_{m}(t)\right]^{T} \text { contains the sources assembly at a time } t .
\end{aligned}
$$

The transfer matrix $\mathbf{A}$ between the sources and the sensors is called the mixing matrix.

$\sigma(t)$ is the noise vector which is modelled as a stationary white noise of zero mean and is assumed to be independent of the sources.

The SOBI method attempts to extract the sources $\mathbf{s}(t)$ and the mixing matrix $\mathbf{A}$ from the observed data $\mathbf{x}(t)$. It relies on the second order statistics and is based on the diagonalization of time-lagged covariance matrices under certain assumptions on the source signals. A detailed description of the SOBI procedure may be found in [4].

When BSS techniques are used in the context of modal identification as reported in [5], the mixing matrix identifies to the modal matrix of the structure and the identified sources correspond to the normal coordinates. In this case, the relative contribution of an identified mode may be estimated by the Modal Contribution Indicator (MCI) as proposed in [6]. 


\section{The hankel matrix and enhanced methods}

Block Hankel matrices play an important role in subspace identification of linear systems [7]. They may also be used for identification of nonlinear systems [8] and for structural damage detection [1]. The data-driven block Hankel matrix is defined in Eq. (3), where $2 i$ is a user-defined number of row blocks, each block contains $m$ rows (number of measurement sensors), $j$ is the number of columns (practically $j=N-2 i+1, N$ is the number of sampling points). The Hankel matrix $\mathbf{H}_{1,2 i}$ is split into two equal parts of $i$ block rows which represent past and future data respectively. Compared to the observation matrix $\mathbf{X}$, the Hankel matrix is built using time-lagged vibration signals and not instantaneous representations of responses. This enables to take into account time correlations between measurements when current data depend on past data. Therefore, the objective pursued here in using block Hankel matrices rather than observation matrices is to improve the sensitivity of the detection method. In the following, the PCA and SOBI methods applied on Hankel matrices are called enhanced PCA (EPCA) and enhanced SOBI (ESOBI) respectively. It is expected that these enhanced methods will be able to provide better information about the dynamics of the system. Because of the way the Hankel matrix is constructed, it is important to note that detection can still be achieved even if only one single sensor is available. In this case, the principal components (PCs) extracted from PCA or the column vectors of the mixing matrix given by SOBI are not anymore representative of the modal subspace (spanned by the mode-shape vectors of the structure). Indeed, the use of a single sensor to construct the Hankel matrix does not allow to extract afterwards spatial information on the structure such as mode-shapes. However, even in this case, the column vectors of the mixing matrix are still dynamic features of the structure as they represent the way in which the different sources (i.e., eigenfrequencies) interact in time at the measurement location.

$$
\mathbf{H}_{1,2 i}=\left[\begin{array}{ccccc}
\mathbf{x}_{1} & \mathbf{x}_{2} & \ldots & \ldots & \mathbf{x}_{j} \\
\mathbf{x}_{2} & \mathbf{x}_{3} & \ldots & \ldots & \mathbf{x}_{j+1} \\
\ldots & \ldots & \ldots & \ldots & \ldots \\
\mathbf{x}_{i} & \mathbf{x}_{i+1} & \ldots & \ldots & \mathbf{x}_{i+j-1} \\
\hline \mathbf{x}_{i+1} & \mathbf{x}_{i+2} & \ldots & \ldots & \mathbf{x}_{i+j} \\
\mathbf{x}_{i+2} & \mathbf{x}_{i+3} & \ldots & \ldots & \mathbf{x}_{i+j+1} \\
\ldots & \ldots & \ldots & \ldots & \ldots \\
\mathbf{x}_{2 i} & \mathbf{x}_{2 i+1} & \ldots & \ldots & \ldots \mathbf{x}_{2 i+j-1}
\end{array}\right] \equiv\left[\frac{\mathbf{x}_{p}}{\mathbf{X}_{f}}\right] \equiv \frac{\text { past }}{\text { " future" }}
$$

\section{Damage index based on the concept of subspace angle}

Matrices $\mathbf{U}$ and $\mathbf{A}$ given respectively by Eq. (1) (PCA) or Eq. (2) (SOBI) span subspaces which characterize the state of the system. Any change in the dynamic behaviour which modifies the state of the system consequently modifies its characteristic subspace. This change may be estimated using the concept of subspace angle [9]. This concept can be seen as a tool to quantify existing spatial coherence between two data sets resulting from observations of a vibration system.

Given two subspaces (each with linear independent columns) $\mathbf{S} \in \mathfrak{R}^{m \times p}$ and $\mathbf{D} \in \mathfrak{R}^{m \times q}(p \geqslant q$ ), the procedure is as follows. Carry out the QR factorizations:

$$
\begin{aligned}
& \mathbf{S}=\mathbf{Q}_{S} \mathbf{R}_{S}\left(\mathbf{Q}_{S} \in \mathfrak{R}^{m \times p}\right) \\
& \mathbf{D}=\mathbf{Q}_{D} \mathbf{R}_{D}\left(\mathbf{Q}_{S} \in \mathfrak{R}^{m \times q}\right)
\end{aligned}
$$

The columns of $\mathbf{Q}_{\mathrm{S}}$ and $\mathbf{Q}_{\mathrm{D}}$ define the orthonormal bases for $\mathbf{S}$ and $\mathbf{D}$ respectively. The angles $\theta_{i}$ between the subspaces $\mathbf{S}$ and $\mathbf{D}$ are computed from singular values associated with the product $\mathbf{Q}_{S}^{T} \mathbf{Q}_{D}$ : 


$$
\begin{aligned}
& \mathbf{Q}_{S}^{T} \mathbf{Q}_{D}=\mathbf{U}_{S D} \Sigma_{S D} \mathbf{V}_{S D}^{T} \\
& \Sigma_{S D}=\operatorname{diag}\left(\cos \left(\theta_{i}\right)\right), \quad i=1, \ldots, q
\end{aligned}
$$

The largest singular value is thus related with the largest angle characterizing the geometric difference between two subspaces.

Changes in the dynamic behaviour of a structure (e.g., damage, onset of nonlinearity) may be detected by monitoring the angular coherence between subspaces estimated from a reference observation set and from an observation set of a current state respectively. A state is considered as reference if the system operates in normal conditions (i.e., absence of damage, low level of excitation). As explained in the previous section, active subspaces which characterize the dynamic behaviour of the system can be built from the first principal components given by EPCA or from the first columns of the mixing matrix obtained by ESOBI.

\section{Examples of industrial applications}

\subsection{Fault detection in electro-mechanical devices}

The first example concerns the case of electro-mechanical devices for which the overall quality at the end of the assembly line has to be assessed.

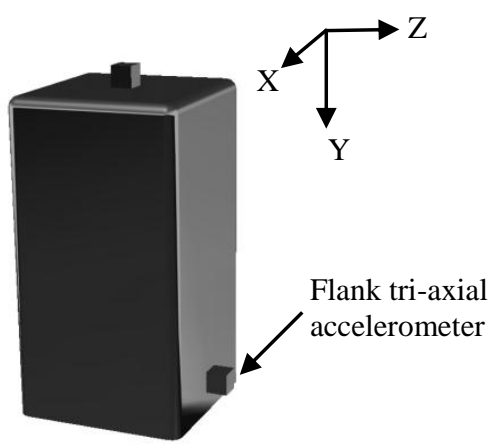

Fig. 1. Location of the tri-axial accelerometer on the flank of the electro-mechanical device.

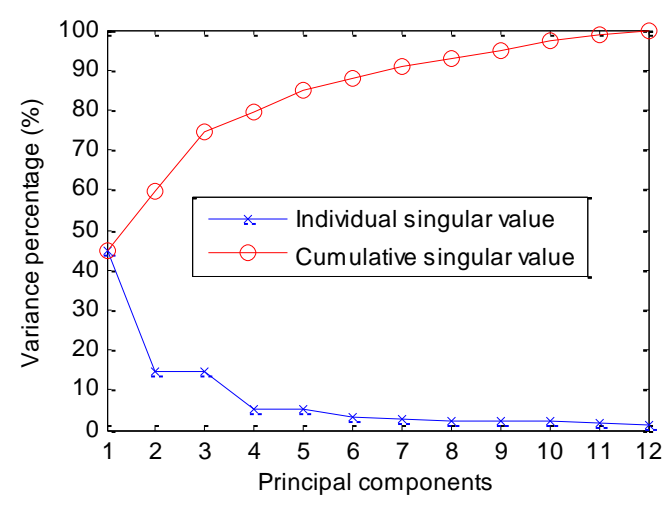

Fig. 2. Diagram of singular value (EPCA).

A set of five good (healthy) devices and four damaged devices was considered. The healthy and faulty devices were determined through productivity and efficiency testing performed in the industry. As this type of quality testing procedure is long and expansive, it is usually performed on a small set of devices at the cost of statistical significance. To speed up the testing procedure while increasing the number of tested devices, alternative methods were sought to characterize the mechanical signature of the device. For this purpose, dynamic responses were collected by a tri-axial accelerometer placed on the flank of the device as illustrated in Fig. 1. Only data measured in one direction on the flank (X, Y or Z in Fig. 1) of the device is used for the detection. As it was found in [10] that fault detection gave the best result when using the data in the $\mathrm{Y}$ direction, only the data in this direction are exploited here to test the proposed methods.

It is worth recalling that using the response measured by one sensor only, detection cannot be performed by subspace methods such as SOBI and PCA. However, when EPCA and ESOBI are performed through the Hankel matrices, it is possible to construct a subspace even if only one response signal is available. For illustration, Fig. 2 shows the relative variances (energies) of the PCs when 12 block rows are used to construct the Hankel matrix for the reference data in the EPCA method. Detection results are shown in Fig. 3 for the whole set of rotating devices 
including the four healthy devices (H1-H4) and the four damaged devices (D1- D4). Figure 3a presents the detection results of EPCA when 4PCs are taken into account, cumulating about $80 \%$ energy of the system (Fig. 2). Similarly, the detection results of ESOBI are given in Fig. 3b. These figures show that both techniques are able to detect accurately all the faulty devices.

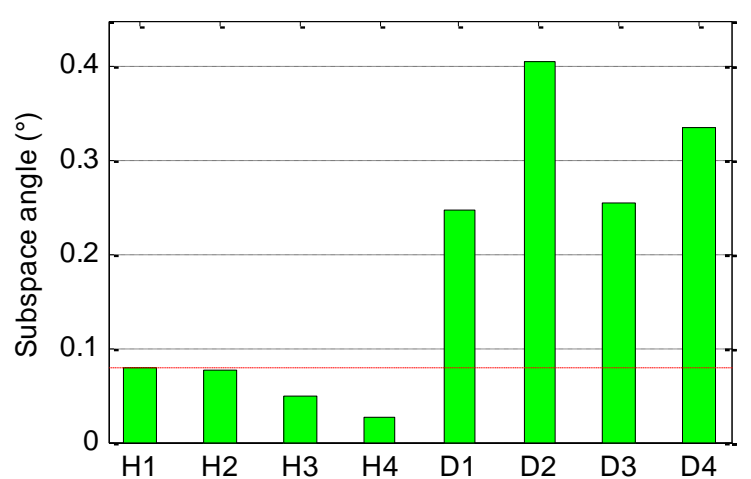

(a) EPCA based-detection

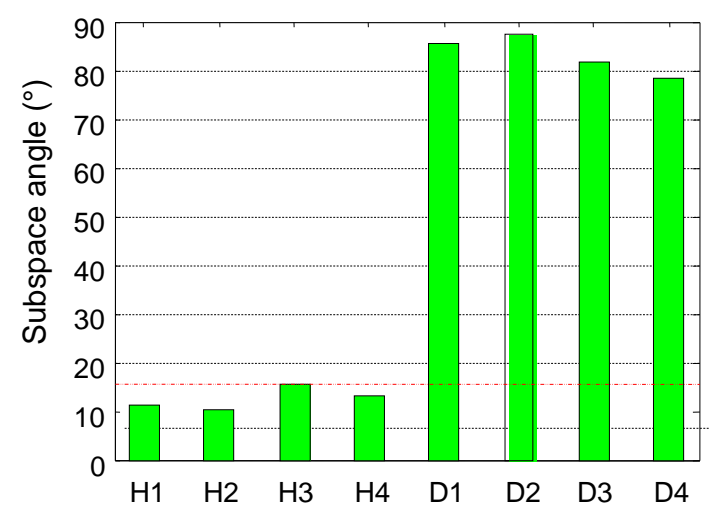

(b) ESOBI based-detection

Fig. 3. Fault detection in electro-mechanical devices by EPCA and ESOBI (The dashed horizontal lines correspond to the maximal values for the healthy devices).

\subsection{Quality control of welded joints}

The second example involves an industrial welding machine located in a steel processing plant. The welding machine was instrumented with a mono-axial accelerometer placed on the forging wheel which is used to flatten the welded joint.

The quality of welds depends on several parameters. For this purpose, six welded joints with nominal welding parameters (OK1-OK6) and 27 joints with out-of-range parameters were studied. The welding parameters that were altered by the machine operator are reported in Table 1: they correspond to covering, compensation, current and forging pressure. From a microscope quality control, welded joints $\mathrm{C}$ and $\mathrm{G}$ were diagnosed good, welded joints $\mathrm{A}$, D, E, H were diagnosed acceptable and welded joints B, F, I were diagnosed bad.

Table 1

Modification of welding parameters (with respect to the nominal parameters)

\begin{tabular}{llcc}
\hline \multicolumn{1}{c}{ Name } & \multicolumn{1}{c}{ Modified parameter } & Number of samples & Microscope quality control of the weld \\
\hline OK & Nominal level & 6 & Good \\
Welding A & $-33 \%$ covering & 3 & Acceptable \\
Welding B & $-66 \%$ covering & 3 & Bad \\
Welding C & $-33 \%$ compensation & 3 & Good \\
Welding D & $-66 \%$ compensation & 3 & Acceptable \\
Welding E & $-10 \%$ current & 3 & Acceptable \\
Welding F & $-20 \%$ current & 3 & Bad \\
Welding G & $-10 \%$ forging pressure & 3 & Good \\
Welding H & $+5 \%$ forging pressure & 3 & Acceptable \\
Welding I & $-66 \%$ covering and compensation & 3 & Bad \\
\hline
\end{tabular}

Detection results by EPCA and ESOBI are given in Fig. 4. Note that for EPCA, four principal components which cumulate most of the energy of the system were considered to define the reference subspace while for ESOBI, only 
two vectors of the mixing matrix were taken as they correspond to the two sources which show the most significant MCI. In this example, ESOBI looks a little more sensitive to damage than EPCA.
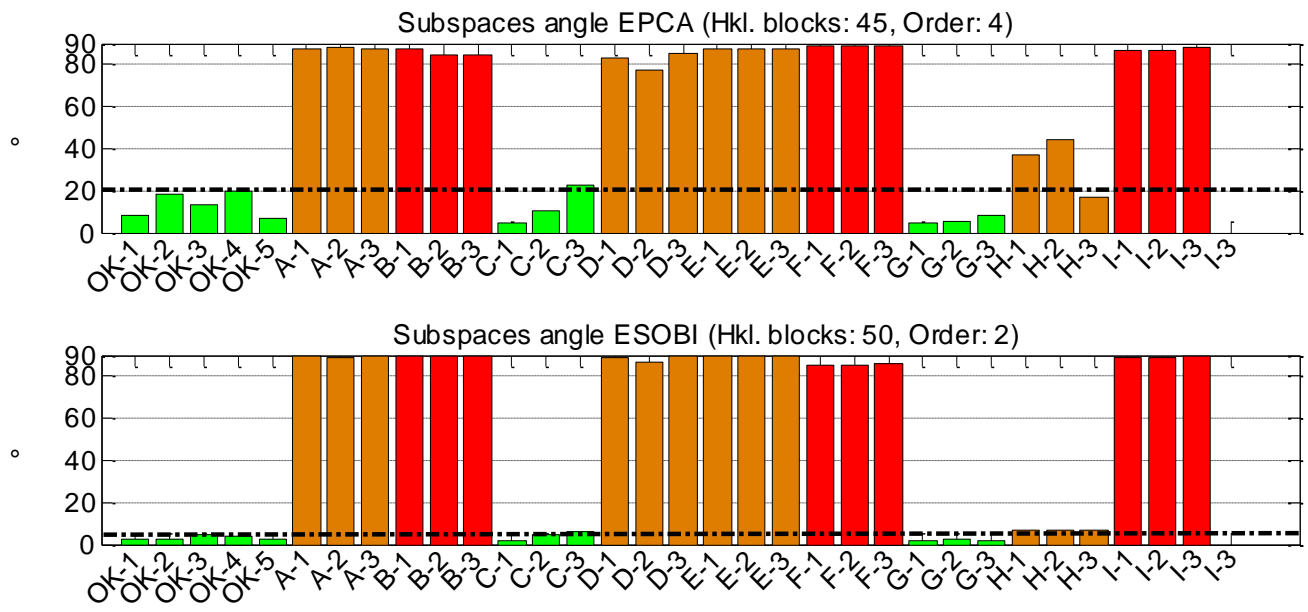

Fig. 4. Damage detection in welds by EPCA (top) and ESOBI (bottom) (the dash-dot lines correspond to the maximal values for the good welded joints).

\section{Conclusion}

A well-known drawback of most BSS techniques is their inability to perform modal analysis when only a small number of sensors are available, which limits their use as damage detection methods based on modal information. However, it is often the case in practice that only one sensor is used for monitoring a machine. In this paper, some alternatives based on the use of Hankel matrices have been presented to overcome this issue. Two methods (EPCA and ESOBI) have been introduced and tested to diagnose faults or to detect damage in industrial applications. In the EPCA method, the order (number of principal components) is determined by looking at the cumulated variance in the singular values diagram, while in the ESOBI method, order selection is based on the Modal Contribution Indicator (MCI) of the sources. Results obtained on the industrial examples show that detection provided by both methods is quite automatic, fast and reliable.

\section{Acknowledgment}

The authors would like to acknowledge the financial support of the "Région Wallonne" of Belgium in the framework of the "MINT" project.

\section{References}

[1] A.-M. Yan and J.-C. Golinval, Null subspace-based damage detection of structures using vibration measurements, Mechanical Systems and Signal Processing 20 (2006), 611-626.

[2] V.H. Nguyen and J.-C. Golinval, Detection of nonlinearity onset based on Kernel Principal Component Analysis, Engineering Structures 32 (2010), 3683-3691.

[3] P. De Boe and J.-C. Golinval, Principal component analysis of a piezosensor array for damage localization, Structural Health Monitoring (2003), 137-144.

[4] A. Belouchrani, K.A. Meraim, J.F. Cardoso and E. Moulines, A blind source separation technique using Second - order statistics, IEEE transactions on signal Processing 45 (1997), 434-444.

[5] F. Poncelet, G. Kerschen, J.-C. Golinval and D. Verhelst, Output-only modal analysis using blind source separation techniques, Mechanical Systems and Signal Processing 21 (2007), 2335-2358. 
[6] S.I. McNeil and D.C. Zimmerman, Blind modal identification applied to output-only building vibration, Proceedings of the IMAC-XXVIII, Florida, USA, February, 2010.

[7] P.V. Overschee and B. De Moor, Subspace identification for linear systems-Theory-Implementation-Applications, Kluwer Academic Publishers, 1997.

[8] S. Marchesiello and L. Garibaldi, A time domain approach for identifying nonlinear vibrating structures by subspace methods, Mechanical Systems and Signal Processing 22 (2008), 81-101.

[9] G.H. Golub and C.F. Van Loan, Matrix computations, Baltimore, The Johns Hopkins University Press, 1996.

[10] C. Rutten, C. Loffet and J.-C. Golinval, Damage detection of mechanical components using null subspace analysis, 2th International Symposium ETE’2009 - Brussels, Belgium. 

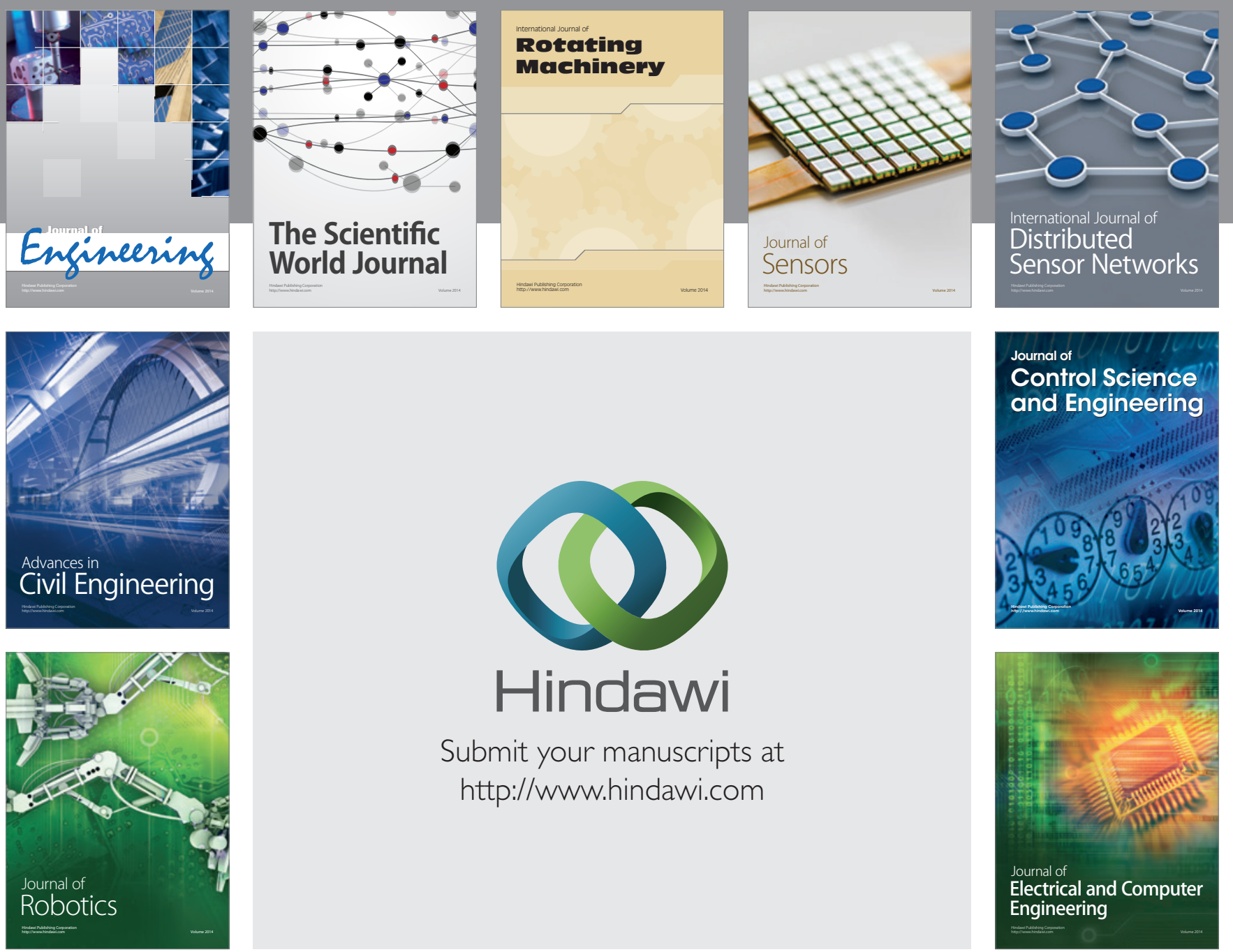

Submit your manuscripts at

http://www.hindawi.com
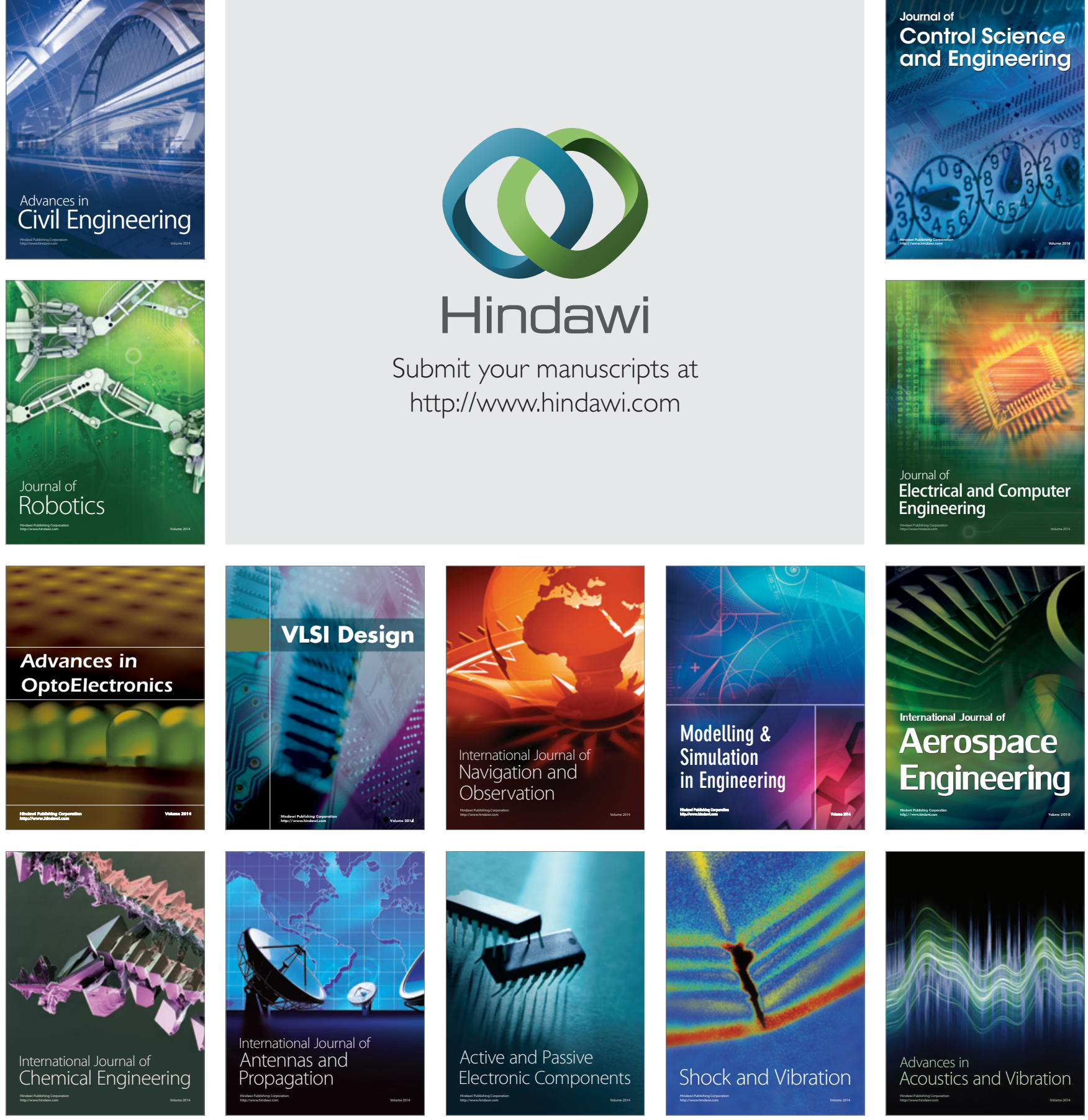